\title{
A Comprehensive Survey of New Different Protection Schemes For Micro Grid System
}

\section{Adinarayana B*}

Siddhartha Institute of Technology and Sciences, Hyderabad, Telangana, India

\begin{abstract}
The emergence of renewable energy with high consumers energy demand has led to the concept of micro-grids in the world in recent years. The development and expansion of micro-girds with renewable energy is facing challenges with the protection needing serious attention. The challenges result from the difficulties in coordinating protection devices. The presence of various energy sources makes the micro-grid protection sensitive to changes in current and voltage levels. A micro-grid operates in parallel or independently with the existing utility network as grid-connected mode or islanded mode, respectively. Whatever the mode of operation, the protection system has to detect short circuits and clear faults to isolate only the faulty portions. In this study, micro-grid protection comprehensive review is performed to discuss the present situation. Protection issues and existing protection schemes for micro-grid are presented based on the previous achievement in the area. This work will contribute in the assessment and implementation of micro-grid protection to provide reliable and safe electricity to consumers.
\end{abstract}

Keywords: Micro grid system; Electric power system; Wireless communication network

\section{Introduction}

With conventional power systems struggling to meet the consumers increasing demand of electric power, renewable energy resources are being installed to mitigate the energy shortfall. Microgrid idea is a way of integrating multiple renewable energy sources as well as conventional sources and energy storage devices and loads into electric power system [1]. The micro-grid operates in the gridconnected mode or it can be disconnected from the utility by the static switch at the point of common coupling (PCC) to operate in autonomous mode, i.e. without grid connection [2]. Micro-grids have the advantages of improved system efficiency, reduced costs, better power quality, and increased system reliability. In spite of the advantages associated with micro-grid, one major challenge it faces is with its protection. The protection and the control challenges are more difficult to handle with the renewable sources intermittence. This is mostly observed with solar, wind, and hydro distributed generations (DGs). Issues such as un-balance between supply and load demand, bi-directional power flow, reverse power flow, low inertia leading to severe frequency deviations in islanded mode of operation, limited short circuit capacity which in return causes distinct reduction in micro-grid fault level $[3,4]$ are part of those challenges. In [5] key protection challenges are discussed with feasible solutions. When a fault occurs on the utility grid, the so-called islanded mode should take place to allow the quick isolation of the micro-grid from the rest of the distribution network. However, if the fault occurs within a zone of the protection scheme, only the faulted zone within the micro-grid should be isolated with limited damage. This is in accordance to the IEEE standard 1547 [6]. In the literature there are diversified strategies that offer feasible and reliable protection schemes for micro-grids. This paper will offer a comprehensive extensive review of the protection schemes currently applied to address micro-grid protection issues in both islanded and grid-connected operating modes. Protection issues are clearly introduced. Existing protection schemes are presented and discussed. The covered protection schemes are precisely termed as adaptive protection, differential protection, distance protection, voltage-based Protection, and coordinated protection techniques. This micro-grid protection review will surely provide the reader a wide and greater understanding of the present topic.

\section{Protection Issues Summary}

As already mentioned micro-grids can work in grid-connected mode or islanding mode. In both modes of operation, the protection scheme has to be reliable. In real applications, generation sources are frequently inverter based when in islanded mode of operation and that is a problem. The reason is inverter fault currents are limited by the rating of devices to nearly two times the rated current [7]. Thus, making use of traditional overcurrent protection techniques is challenging with induced low magnitudes of fault current. The protection scheme strategies should be identical for either mode of operation as a general principle. In case of high penetration of DGs units that operate through synchronous or induction generator, changes in fault current level restricted to a smaller value is a consequence. Traveling wave-based protection scheme for inverter-dominated micro-grid using mathematical morphology is proposed in Li X et al. [8]. When the fault current is below the load current, some relays may not trip, while others react to fault with a time delay. The potential unnoticed fault current could spreads out into the system and damage equipment [7]. Expansion of the traditional overcurrent protection techniques is relevant. For a stable micro-grid, active and reactive power balance with the power being consumed by the loads is eminent. In that way, while ensuring active and reactive generation control, voltage and frequency must remain within pre-defined limits [9].

\section{Existing Protection Schemes}

Micro-grids protection schemes can be classified as: voltage-based protection, adaptive protection, and differential protection, distance

*Corresponding author: Adinarayana B, Associate Professor, Siddhartha Institute of Technology and Sciences, Hyderabad, Telangana, India, Tel: 095426 64980; E-mail: ravimtech2@gmail.com

Received June 12, 2018; Accepted August 29, 2018; Published September 05, 2018

Citation: Adinarayana B (2018) A Comprehensive Survey of New Different Protection Schemes For Micro Grid System. J Electr Electron Syst 7: 270. doi: $10.4172 / 2332-0796.1000270$

Copyright: $\odot 2018$ Adinarayana B. This is an open-access article distributed under the terms of the Creative Commons Attribution License, which permits unrestricted use, distribution, and reproduction in any medium, provided the original author and source are credited. 
pro-tection, over current protection [10], techniques with external devices utilization [11], the coordinated protection techniques and so on. Depending upon the micro-grid system complexity, multiple protection strategies can be used at the same time. Importantly,

These techniques must comply with the above mentioned requirementis in regard of grid-connected mode and islanded mode of operations with fault inside or out of the micro-grid.

\section{Adaptive Protection Scheme}

In adaptive protection scheme, an automatic readjustment of relay settings occurs when micro-grid changes from grid connected mode to islanded mode and vice versa. The adaptive protection scheme is an online system that can modify the desired protective response to change under system conditions or requirements in an appropriate manner by the use of external control actions or generated signals. This protection scheme makes use of numerical directional over current relays (DOCRs) for its technical requirements and for practical implementation. For efficient protection, the use of communication systems and standard communication protocol is needed to enable relays to communicate and exchange information with a central computer or between different relays. In 2013 Ustun et al. [12] developed an adaptive micro-grid protection scheme that utilizes the benefits of bi-directional communication capability of the smart grid. Simulations were conducted using OPNET to examine the message latency for the proposed scheme over a WiMAX-based wireless communication network. Results show that the proposed scheme is able to operate over an integrated smart grid communication network. In 2014 Voima et al. [11] presented an adaptive protection scheme. Their results showed that distance protection can perform well in island operation. They observed that directional overcurrent may fail to operate selectively in island operation due to the clear margin with maximum load current and fault currents. The proposed scheme has the advantage of maintaining selective operation after topology changes. Lin et al. [13] proposed a phasor measurement unit-based distance protection strategy for micro-grids in distribution system. The results show that the adaptive distance protection methodology has good selectivity and sensitivity. They identified that compared to adaptive overcurrent protection and differential protection, this adaptive distance protection methodology has almost the same investment. However, it has better capability on monitoring and controlling the whole system with highresolution real-time measurement. In 2016 Rana et al. [14] presented a protection scheme, which employs a centralised secure real-time monitoring infrastructure and control method that achieves the accurate state estimation and a desired control performance. The results obtained showed that the system can mitigate cyber-attacks and stabilise the system. In Lin $\mathrm{H}$ et al. [15], the authors proposed an adaptive overcurrent protection scheme based on synchronous phase measurement for different operation modes in micro grid. The achieved test results show that with the assistance of estimation model, one can modify protective devices settings for a new operation state accurately and intelligently. They stated that with the deployment of modern communication and measurement systems, the protection scheme can modify the protective devices settings intelligently and automatically in large distribution systems. Adaptive protection scheme for smart micro-grid with electronically coupled distributed generations is recently proposed in Sitharthan $\mathrm{R}$ et al. [16]. The research reveals that by coupling electronically distributed energy resources and, in particular, renewable energy sources, irrespective of the operating mode of the micro-grid, the method is capable of monitoring the micro-grid and instantly updates relay fault current according to the variations that occur in the system to assure increase in micro-grid consistency. In Dang $\mathrm{K}$ et al. [17] an adaptive protection method for inverter dominated micro-grid, which adaptively selects the different fault detection methods in grid-connected and islanded modes to provide selectivity and re-liability improvement in micro-grid protection is discussed. The technical challenges associated with adaptive protection scheme are related to but not limited to, short life cycles as a result of fast changing software technology, relays replacement high-cost, fast and reliable communication links involving high cost, Complexity in design, which call for numerous intelligent technologies, necessity to plan all possible configurations of the micro-grid before implementing the required protection.

\section{Differential Protection Scheme}

The principle of the differential protection is that it compares the currents entering and leaving a chosen protected zone and operates when the difference between these currents exceeds a predeter-mined magnitude. That is, the differential protection is a basic ap-plication of Kirchhoff's current law. Recently, Gururani et al. [18] proposed a micro-grid protection using Hilbert-Huang transform-based differential scheme. In 2013 Ustun et al. presented communication assisted protection scheme for micro-grids environments that employ differential current as the protection scheme. This protection scheme has the advantage of micro-grid central protection unit, which eliminates some drawback of the differential current protection and has the ability to monitor the entire micro-grid to be able to choose different restraint current calculation algorithms. In addition, the corresponding scheme proposed a suit-able back-up protection scheme, which operates under communication failures. A time-frequency transform-based differential scheme for micro-grid protection is discussed. Sortomme et al. introduced a differential protection scheme to protect microgrids with islanding capability. That differential protection scheme works by comparing current measurements from several devices on the extremities of the designed protection zones. This method is effective in detecting micro-grid faults presence as digital relay differential protection schemes at significantly lower costs. A case study showed that the optimal placement solution could reduce investments by $>50 \%$ when compared to having a relay at each line segment. In 2014 Ranjbar et al. proposed a comprehensive differential protection scheme utilising communication links to protect medium-voltage (MV) microgrids. The protection scheme employs differential current to isolate the fault, and can address protection issues of micro-grid in both modes of operation. A backup protection scheme was also proposed in the event of communication failure. They stated that the use of programmable digital relays will be not only able to protect the micro-grid, but also help enhance communication to other parts of the system. In $2015 \mathrm{Kar}$ et al. proposed a protection strategy, which functions with different modes and topologies of micro-grid. The scheme derives its differential features at respective feeders further used to build data-mining models, which are used for a final relaying decision. The proposed data-mining model was extensively tested on the standard IEC micro-grid model and provided performance accuracy of $99 \%$ with a relaying speed of less than one and half cycle. The performance comparison with existing relays indicates that the proposed scheme can provide a highly reliable and effective protection measure for the micro-grid. Abdul wahid et al. proposed a differential protection scheme using a fuzzy system with Hilbert-space-based power theory (FHSP). Implementation of this protection scheme uses the power difference between the sending and receiving ends of the feeder to distinguish faults conditions. This protection scheme has the advantage of operating in less than two 
cycles after the occurrence of the fault and its error detection is not dependent on the selection of threshold values. The algorithm operates correctly for all types of faults whilst preventing unwanted tripping, even in case of distorted data due to current transformer saturation or data mismatches. The technical challenges associated with differential protection scheme are related to but not limited to, communication system high-cost, failure of the communication system, problems due to transients during connection and disconnection of DGs.

\section{Distance Protection Scheme}

Micro-grid distance protection uses impedance measurements to effectively detect faults. It is able to isolate a fault that happens on either side of the protected circuit since it also has the ability to operate in the case of reverse faults but reach settings must be different for forward and reverse faults. In 2015 Lin et al. adopted distance protection for one mid-voltage level micro-grid. The results showed that the variations of the fault currents seen by the forward relays are much larger than the backward relays. On the other hand, the fault currents experienced almost no change except with intermittence of renewable energy. In fact, it is shown that the distance protection scheme had a satisfactory performance to clear the various faults. Habib et al. proposed a distribution line protection strategy utilizing contemporary communication technologies between multi-agents. The scheme studies the variations in phase currents at both ends of the distribution line to accurately identify and isolate the faulted section in the system. The results of the simulations showed strong indications to the effectiveness of the proposed protection scheme. The technical challenges associated with distance protection scheme are related to but not limited to inaccuracy in extracting non-distorted currents, protection under reach, and difficulties to measure the admittance for short lines.

\section{Voltage-based protection scheme}

Voltage-based protection uses measurements of voltage to protect power systems against numerous faults. Voltage-based protection protects power systems against both in and out of zone faults circumstances. A communication link is required in the scheme to categories which faults are in-zone or out-of-zone. In 2013 Ndou et al. evaluated the performances of an under/over voltage relay and that of a rate of change of frequency (ROCOF) relay. The protection schemes are compared for different fault types and locations. They used MATLAB/ SIMULINK software to build the test network with its protection schemes. The results showed that the ROCOF relay, which was blinded to some grid faults, has a lower performance than an under/over voltage relay. In 2016 Bui et al. introduced uni-grounded low-voltage (LV) protection scheme for micro-grid configurations. The protection structure consists of six individual protection zones in a uni-grounded LV AC micro-grid. For each protection zone, possible fault protection methods are proposed, which can be used for both main and back-up protections of a uni-grounded micro-grid. Operators of the microgrid can properly select which main and back-up protection methods are optimal. In 2017 Zarei et al. introduced a comprehensive digitalrelay-based protection for micro-grid. The method is independent of the micro-grid operation mode and, efficiently, protects lines, DGs, and PCC, while benefiting from single-phase tripping. The results assured characteristics of safety, selectivity, and reliability in the operation of protective devices in both modes of operation for microgrid internal and external faults. Monadi et al. presented a centralized protection scheme for MV DC micro-grids. The proposed scheme uses a communication-assisted fault detection method with a centralised protection coordinator. A fault isolation technique that provides economic, fast, and selective protection is considered. The protection scheme is supported by a backup pro-tection that is activated in case of communication failure. The technical challenges associated with voltage-based protection scheme are related to but not limited to protection devices malfunction causing voltage drop, tendency to depend on the micro-grid configuration, and less sensitivity in gridconnected mode of operation.

\section{Coordinated Protection Technique}

Protection schemes are coordinated to enhance efficiency in operations of primary and backup lines of defence. Primary protection schemes acts as the first line of protection against faults, while backup protection schemes acts in the event of primary protection failure. This process makes the protection scheme more resilient and reliable in operation. Haron et al. presented a protection strategy with systematic coordination approach using combination of three types of protection termed as overcurrent, directional, and differential protection. They found that although over current relays are known for superiority in protection of radial distribution system, the same relays are found incapable to protect a network with some distributed generators inside the grid. Their investigation results proved that addition of DOCRs could help to improve the network security but dependency is insufficient to isolate the smallest faulted area possible. Further tests show that by combining overcurrent (OC), directional overcurrent (DOC), and differential protection relays, it is possible to successfully protect the micro-grid with topology changes. Sahoo presented a protection scheme to address the existing protection challenges encountered due to the presence of both non-inverter interfaced DGs and inverter inter-faced DGs in the same micro-grid. The proposed protection scheme used DOCRs for a micro-grid that consists of distributed energy resources such as photovoltaic arrays, wind, diesel generator, and micro hydro-turbine for various fault locations. In 2016 Sharaf et al. proposed the use of communication assisted dual setting DOCRs for micro-grid protection considering grid connected mode and islanded mode of operation. The proposed scheme has been compared against the usual conventional directional overcurrent relay approach. The results show that the pro-posed approach overcomes the problem of infeasibility experienced by the conventional scheme, without the need for in-stalling fault current limiters. Furthermore, the protection coordination strategy decreases the relays operating times and maintains adequate protection coordination among the relays. In 2017 Saleh et al. considered the N-1 contingency to determine the optimal relay settings to solve a protection coordination problem in micro-grid. In this scheme, the optimal DOCRs settings for a microgrid are determined considering all network topologies in gridconnected and islanded topologies. The proposed approach is tested on a 9-bus radial distribution system and on the IEEE 14-bus meshed distribution system, both equipped with DGs units. The results show that DOCRs can be optimally set for micro-grids to accommodate multiple network configurations under various contingencies. The challenge with the coordinated protection techniques is concerned mainly with the calculation of protection devices optimal settings and the dependence on a communication system.

\section{Conclusion}

Several protection schemes for micro-grids were reviewed in this paper. To contribute in the proliferation of micro-grids this paper provided a review on the existing protection schemes. Microgrids have the potential to meet reliability and power quality. It is 
demonstrated that micro-grids protection area needs serious attentions because the operation of micro-grids in both grid-connected mode and islanded mode depends on the existing protection strategies. In fact, numerous protection schemes have been introduced in the literature and some important references are mentioned in this work. Adaptive protection, differential protection, distance protection, voltage-based protection, and the coordinated protection for micro-grids were discussed extensively. Finally, de-pending on the type of micro-grid and considering the constraints, a successful protection scheme should have the capability to protect the micro-grid against all types of faults.

\section{References}

1. Nikmehr N, Ravadanegh SN (2016) Reability evaluation of multi-microgrids considering optimal operation of small scale energy zones under loadgeneration uncertainties. Int J Electr Power Energy Syst 78: 80-87.

2. Najy WKA, Zeineldin HH, Woon WL (2013) Optimal protection co-ordination for microgrids with grid-connected and islanded capability. IEEE Trans Ind Electron 6: 1668-1677.

3. Zeineldin H, Mohamed YA-RI, Khadkikar V, Pandi VR (2013) A protection coordination index for evaluating distributed generation impacts on protection for meshed distribution systems. IEEE Trans Smart Grid 4: 1523-1532.

4. Li Y, Gao W, Jiang J (2014) Stability analysis of microgrids with multiple DER units and variable loads based on MPT. IEE PES General Meeting Conf. Exposition, Maryland, USA, 27: 1-5.

5. Sharkh SM, Abu-Sara MA, Orfanoudakis GI, Hussain B (2014) Power electronic converters for microgrids. $1^{\text {st }}$ edn. John Wiley \& Sons, Singapore.

6. Basso TS, DeBlasio R (2004) IEEE 1547 series of standards: interconnection issues. IEEE Trans. Power Electron 19: 1159-1162.

7. Almutairy I (2016) A review of coordination strategies and techniques for overcurrent challenges to microgrid protection. Saudi Arabia Smart Grid (SASG), Jeddah, Saudi Arabia, pp: 1-4.
8. Li X, Dysko A, Burt GM (2014) Traveling wave-based protection scheme for inverter-dominated microgrid using mathematical morph-ology. IEEE Trans Smart Grid 5: 2211-2218.

9. Salam AA, Mohamed A, Hannan MA (2008) Technical challenges on microgrids. J Eng Appl Sci 3: 64-69.

10. Mirsaeidi S, Said DM, Mustafa MW, Ghaffari K (2014) Review and analysis of existing protection strategies for microgrids. J Electr Syst 10: 1-10.

11. Voima S, Laaksonen H, Kauhaniemi K (2014) Adaptive protection scheme for smart grids. 12th IET Int Conf Developments in Power System Protection, Copenhagen, Denmark.

12. Ustun TS, Khan RH, Hadbah A, Kalam A (2013) An adaptive microgrid protection scheme based on a wide-area smart communications network. IEEE Latin America on Communications, Santiago, Chile.

13. Lin H, Guerrero JM, Vasquez JC, Liu C (2015) Adaptive distance protection for microgrids. Proc 41st Annual Conf Industrial Electronics Society, Yokohama Japan, pp: 00725-00730.

14. Rana MM, Li L, Su SW (2016) Microgrid protection and control through reliable smart grid communication systems. 14th Int Conf Control Automation Robotics \& Vision, Phuket, Thailand.

15. Lin H, Guerrero JM, Jia C, Tan Z, Vasquez JC, et al. (2016) Adaptive overcurrent protection for microgrids in extensive distribution systems. Proc 42th Annual Conf. Industrial Electronics Society, IECON 2016, Florence, Italy, pp: 4042-4047.

16. Sitharthan R, Geethanjali M, Senthil Pandy TK (2016) Adaptive protection scheme for smart microgrid with electronically coupled distributed generations. Alexandria Eng J 55: 2539-2550.

17. Dang K, He X, Bi D, Feng C(2011) An adaptive protection method for inverte dominated microgrid. Int. Conf. Electrical Machines and Systems, Beijing, China, pp: $1-5$

18. Gururani A, Mohanty SR, Mohanta JC (2016) Microgrid protection using Hilbert-Huang transform based-differential scheme. IET Gener Transm Distrib 10: $3707-3716$. 